\title{
Bioinformatics analysis and verification of key genes associated with recurrent respiratory tract infections
}

\author{
XIANG JIN $^{1}$, ZHIYONG JI ${ }^{2}$, XIAODAN LI ${ }^{1}$, RUI LIU ${ }^{1}$ and YINGHUI GUAN ${ }^{1}$ \\ Departments of ${ }^{1}$ Respiratory Medicine and ${ }^{2}$ ICU, The First Hospital, \\ Jilin University, Changchun, Jilin 130000, P.R. China
}

Received November 23, 2016; Accepted February 2, 2018

DOI: $10.3892 / \mathrm{ijmm} .2018 .3640$

\begin{abstract}
We evaluated the key genes related with recurrent respiratory tract infections (RRTIs), and then elucidated the possible molecular mechanisms of RRTIs. Neutrophil was isolated from peripheral bloods of the recurrent lower respiratory tract infection patients and healthy volunteers, respectively. The next generation sequencing information was obtained after RNA extraction, purification, library construction and sequencing. The sequencing information was preprocessed. Bioinformatics analysis including analysis of differentially expressed genes (DEGs), Gene Ontology (GO) and pathway enrichment analysis, protein-protein interaction (PPI) analysis and transcription factors analysis were performed. The key genes were verified by real-time PCR. In total, 17 significant DEGs were obtained in case group compared with the control group by bioinformatics analysis. Then, 6 of 17 genes were detected by real-time PCR. There was statistical significance between case and control groups for peroxisome proliferator-activated receptor- $\gamma$ (PPARG), prostaglandin-endoperoxide synthase 2 (PTGS2), transferrin $(\mathrm{TF})$ and interleukin-10 (IL-10) $(\mathrm{P}<0.05)$, and there was no statistical significance between case and control groups for TIMP metallopeptidase inhibitor 1 (TIMP1) and matrix metallopeptidase 1 (MMP1). PPARG, PTGS2, TF and IL-10 are key genes associated with the progression of RRTIs. We speculate that TIMP1 and MMP1 may also be involved in the progression of RRTIs, but further studies with large number of samples are needed for verification.
\end{abstract}

\section{Introduction}

Recurrent respiratory tract infections (RRTIs) are not uncommon in children in the 1st year of life, mainly caused

Correspondence to: Dr Yinghui Guan, Department of Respiratory Medicine, The First Hospital, Jilin University, 71 Xinmin Street, Changchun, Jilin 130000, P.R. China

E-mail: yinghuig2016vip@163.com

Key words: recurrent respiratory tract infections, differentially expressed genes, bioinformatics analysis, real-time PCR by immaturity of the immune system and exposure to pathogens (1). It is said that a person diagnosed with RRTIs should meet at least one of the following criteria: not less than 6 respiratory infections per year; not less than 3 respiratory infections per year involving the lower airways or not less than 1 respiratory infection per month involving the upper airways from September to April (2). Up to $25 \%$ of children $<1$ year of age and $\sim 18 \%$ of children aged 1 to 4 years suffer from RRTIs in developed countries (3). It occurs both in children and adults $(4,5)$. Thus, early diagnosis of RRTIs and understanding of its pathogenesis are urgently needed.

Some prevention strategies for RRTIs including the use of immunostimulants and vaccines and the reduction of risk factors have been developed (1). One study indicated that treated with vitamin $\mathrm{D}_{3}$ may be able to reduce disease burden of patients with frequent respiratory tract infections (6). Pleuran ( $\beta$-glucan from Pleurotus ostreatus) has a potential anti-allergic effect for children with RRTIs (7). Human milk probiotic Lactobacillus fermentum CECT5716 may be useful for the prevention of upper respiratory tract infections in infants (8). De Benedetto and Sevieri showed that OM-85 could decrease exacerbation frequency of respiratory tract infections in children and adults at risk (5). Though many prevention and treatment measures have been found, the molecular mechanisms of RRTIs are not clear. Understanding of the molecular mechanisms can help us find more effective treatment for RRTIs.

In the process of infection and anti-infection, innate immune system, which serves as the first line of defense, plays the roles of limiting the spread of infection and reducing the tissue damage caused by the inflammatory reaction (9). As a major member of the innate immune system, neutrophil plays important parts in that process. In this present study, peripheral blood neutrophil was used to study the possible molecular mechanisms of RRTIs. We first used the bioinformatics methods to find the key genes associated with RRTIs. Then, the real-time PCR was used to verify the results of bioinformatics. We aimed to find the key genes related with RRTIs, and then elucidate the possible molecular mechanisms of RRTIs.

\section{Materials and methods}

Samples. Fresh peripheral blood was obtained from 9 patients with recurrent lower respiratory tract infection and 9 healthy 
controls with $5 \mathrm{ml}$ for every case. The demographic and clinical characteristics of subjects are shown in Table I. The mean age of patients was 51.5, and the mean age of healthy controls was 54 . Heparin $(10 \mathrm{U} / \mathrm{ml})$ was used to exert anticoagulant effect for blood, and then the blood was stored at $4^{\circ} \mathrm{C}$. Informed consent was obtained from subjects before blood sample collection. The present study was approved by Ethics Committee of First Hospital, Jilin University (no. 2014-078). Single RNA sample was not up to the requirements of sequencing, thus 3 cases of blood samples were extracted and mixed into 1 sample for sequencing. Therefore, the samples included 3 recurrent lower respiratory tract infection samples and 3 control samples. Neutrophil was isolated from peripheral bloods of the recurrent lower respiratory tract infection patients and healthy control samples, respectively.

Processing and quality assessment of raw data. The next generation sequencing information was obtained after RNA extraction, purification, library construction and sequencing, and was stored in a FASTQ file. The quality of the data was assessed using FastQC v0.11.4 (http://www.bioinformatics. babraham.ac.uk/projects/fastqc). Base content distribution was used to detect whether there was AT, GC separation in sequencing data, and AT, GC separation could affect the results of subsequent bioinformatics analysis. GC content distribution was mainly used for detecting whether the GC distribution of the data was normal. Sequence base quality was used to detect the average quality of the sequencing data.

The sequencing data included some adapters and low quality reads, and these sequences could cause a great deal of interference for subsequent analysis. Thus, the removal of adapter sequences and quality filtering were performed. The adapter sequences at least $10 \mathrm{bp}$ overlap at the $3^{\prime}$ end were removed with cutadapt (version 1.2.1) (10), and the quality filtering was performed by $5 \mathrm{bp}$ window method with average quality score of window $\geq Q 20$, the length at least $50 \mathrm{bp}$ and no uncertain bases ('N') in sequences.

Reference genome information and comparative analysis. The sequencing data was compared to the genome. The comparative analysis was performed with Bowtie2 2.1.0/TopHat2 2.1.0 (http://tophat.cbcb.umd.edu/). The reference genome index was constructed by Bowtie2, and then reads after filtering were compared to the reference genome by using TopHat 2 software.

Standardization of gene expression. The read count compared to every gene and obtained by HTSeq 0.6.1p2 (http://wwwhuber.embl.de/users/anders/HTSeq) was regarded as original expression of genes. The standardization of gene expression was carried out with FPKM (fragment per kilo bases per million fragments).

Analysis of differentially expressed genes (DEGs). edgeR (http:// www.bioconductor.org/packages/release/bioc/html/edgeR.html) is a Bioconductor software package used to analyze the differential expression of repeated counting data. edge $\mathrm{R}$ was used for the detection of differential expression in this study. The read count was preprocessed with TMM normalization provided by limma in $\mathrm{R}$ package $(11,12)$, and the preprocessed data was transformed into gene expression matrix with voom (13). $\log _{2} \mathrm{FCl}>1$ and
Table I. The demographic and clinical characteristics of the subjects.

\begin{tabular}{lcc}
\hline Paremeters & Patients & HC \\
\hline $\begin{array}{l}\text { Number } \\
\text { Age (years) }\end{array}$ & $51.5(32-76)$ & 9 \\
mean (range) & & $54(15-76)$ \\
Sex, $\mathrm{M} / \mathrm{F}$ & $5 / 4$ & $5 / 4$ \\
WBC $\left(x 10^{9} / \mathrm{l}\right)$ & $10.86(4.43-25.9)^{\mathrm{a}}$ & $7.20(3.35-12.13)$ \\
PLT $\left(x 10^{9} / \mathrm{l}\right)$ & $175.6(103-479)^{\mathrm{a}}$ & $244.6(187.3-295)$ \\
D-dimer $(\mu \mathrm{g} / \mathrm{l})$ & $448.7(155-742.9)^{\mathrm{a}}$ & $129.1(74.9-238)$ \\
PCT $(\mu \mathrm{g} / \mathrm{ml})$ & $1.07(0.51-2.06)^{\mathrm{a}}$ & $0.06(0.01-0.3)$ \\
$\mathrm{CRP}(\mathrm{mg} / \mathrm{l})$ & $37.3(20.0-87.7)^{\mathrm{a}}$ & $3.22(0.71-10.02)$ \\
\hline
\end{tabular}

M, male; F, female; WBC, white blood cell; PLT, platelet; PCT, procalcitonin; CRP, C-reactive protein. ${ }^{\mathrm{a}} \mathrm{P}<0.05$ vs. the $\mathrm{HC}$.

P-value $<0.05$ were used as cut-off criterion. Then, the heat map of DEGs was drawn using gplots in R package (14).

Gene Ontology (GO) and pathway enrichment analyses. GO is a tool used for gene annotation by collecting defined, structured, controlled vocabulary, mainly including 3 categories, molecular function (MF), biological process (BP) and cellular component (CC) (15). GO slim is a simplified version of GO, only including a part of GO. GO is used for a general understanding of the content of Ontology. Kyoto Encyclopedia of Genes and Genomes (KEGG) is a database used to put associated gene sets into each of their pathway.

We made GO annotation (http://www.bioconductor.org/ packages/release/bioc/html/RamiGO.html), GO slim annotation [OWLTools (Map2Slim) (https://github.com/owlcollab/ owltools/wiki/Map2Slim)] and KEGG pathway enrichment analyses for DEGs. Fisher's exact test was used to calculate the P-values. The P-value cut-off was 0.05 for $\mathrm{GO}$ analysis and $\mathrm{P}$-value was 0.1 for KEGG analysis.

Protein-protein interaction (PPI) network analysis. Search Tool for the Retrieval of Interacting Genes (STRING) (16) database can provide information on the predicted and experimental interactions of proteins. The prediction method of this database came from neighborhood, gene fusion, co-occurrence, co-expression experiments, databases and textmining. The input gene sets were DEGs, and the species was Homo. PPI score was set to 0.4 , and all the protein nodes interacted with each other were DEGs. PPI networks were constructed with Cytoscape software (17).

Analysis of key nodes in network. Four methods including degree centrality (18), betweenness centrality (19), subgraph centrality (20) and closeness centrality (21) were used to study key genes. The scores that network nodes obtained in the 4 methods were observed.

Cytoscape plug-in CytoNCA (parameter setting, network without weight) (22) was used to calculate network centrality. The higher the degree value and the subgraph value of the 
node are, the more important the nodes are in the network. The higher the betweenness values are, the greater the impact of the node in the network is. The higher closeness value indicates that the node is more closely related to the other nodes.

Nodes with the higher scores in 4 methods were predicted as key genes, and clustering effect of these key genes was observed by combining with gplots package.

Analysis of pathways enriched by key genes. KEGG pathways significantly enriched by the key genes were analyzed with $\mathrm{R}$ package clusterProfiler (23). The $\mathrm{P}$-value was adjusted by Benjamini-Hochberg (BH) (24), and pathways with adjusted $\mathrm{P}$-value $<0.05$ were selected.

Analysis of transcription factors of key nodes. We used Cytoscape plug-in iRegulon (25) to analyze transcription factors of key genes. IRegulon integrated some transcription factor databases (Transfac, Jaspar, Encode, Swissregulon and Homer) information, and predicted transcription factors through calculating transcription factor and gene binding motif enrichment analysis. Multiple position weight matrix (PWM) was used in motif enrichment analysis, then sort and score was carried out, and the preferred motif was used to predict the final transcription factor. Parameter setting was minimum identity between orthologous genes $=0.05$, and maximum false discovery rate on motif similarity $=0.001$. The output result was normalized enrichment score (NES). The higher the scores were, the more reliable the results were. The transcription factor and target gene pairs with NES $>3.5$ were selected.

Verification of gene expression. According to both the centrality score of 17 key genes and transcription factor regulation network of key nodes, the prostaglandin-endoperoxide synthase 2 (PTGS2), peroxisome proliferator-activated receptor- $\gamma$ (PPARG), transferrin (TF), interleukin-10 (IL-10), TIMP metallopeptidase inhibitor 1 (TIMP1) and matrix metallopeptidase 1 (MMP1) were chosen for validation.

Neutrophil was isolated from peripheral blood of the recurrent lower respiratory tract infection patients (6 cases) and healthy volunteers (6 cases), respectively. RNA was isolated from peripheral blood by using RNAiso plus (9109; Takara, Tokyo, Japan), RNA was reversely transcribed into cDNA by using PrimeScript ${ }^{\mathrm{TM}}$ RT Master Mix (RR036A; Takara), and the experiment was performed according to the manufacturer's instructions. Real-time PCR was carried out with the help of SYBR-Green kit. The real-time PCR program started with $3 \mathrm{~min}$ of incubation at $50^{\circ} \mathrm{C}, 3 \mathrm{~min}$ at $95^{\circ} \mathrm{C}$, followed by 40 cycles of $10 \mathrm{sec}$ at $95^{\circ} \mathrm{C}$ and $30 \mathrm{sec}$ at $60^{\circ} \mathrm{C}$. After that, a melting curve was constructed for verification of specificity of PCR products by increasing the temperature from 60 to $95^{\circ} \mathrm{C}$ for increment $0.5^{\circ} \mathrm{C}$ for $10 \mathrm{sec}$.

The forward and reverse primers for each gene were designed as follows: 5'-TCGATGCTGCTCTTTCTGAG-3' and 5'-GATAACCTGGATCCATAGATCGTT-3' for MMP1; 5'-CTCGTCATCAGGGCCAAGTT-3' and 5'-GTAGGTCTT GGTGAAGCCCC-3' for TIMP1; 5'-GCCGTGGCCGCAGA TTT-3' and 5'-TGGCATCTCTGTGTCAACCA-3' for PPARG; 5'-ACACAGTCTTCTCATCACTTCGTTT-3' and 5'-AATAG CAGTCCTGAGCTGAGGTTTA-3' for PTGS2; 5'-GTCTAC ATAGCGGGCAAGT-3' and 5'-TTCCAGCCAGCGGTTCT-3' for TF; 5'-TGGAGGACTTTAAGGGTTAC-3' and 5'-TGATG TCTGGGTCTTGGTT-3' for IL-10; 5'-TGACAACTTTGGT ATCGTGGAAGG-3' and 5'-AGGCAGGGATGATGTTCTG GAGAG-3' for GAPDH.

All the data were expressed as mean \pm SEM, and were made into tables. Statistical analysis method was t-test and the software used for data analysis was GraphPad Prism (GraphPad Software, Inc., San Diego, CA, USA). P<0.05 was considered to be statistically significant.

\section{Results}

Processing and quality assessment of raw data. The results for single base quality distribution, base content distribution, GC content distribution and sequence base quality distribution are shown in Fig. 1.

DEGs analysis. After processing of the raw data, 35,663 genes were obtained to select DEGs. The gene was detected in at least one sample. The heat map was standardized data, the difference of expression was large for different genes in sequencing data, and thus it was not appropriate to use raw score. Then, in total 866 DEGs including 438 upregulated and 428 downregulated genes were obtained in case group compared with control group. The heat map and volcano plot are shown in Fig. 2.

GO and pathway enrichment analyses. The results of GO slim and KEGG pathway enrichment analyses are shown in Fig 3. GO-BP (Fig. 3A) was mainly enriched in epithelium development, cell motility, programmed cell death and localization of the cell. GO-CC (Fig. 3B) was mainly enriched in intrinsic component of the membrane, and the membrane parts. GO-MF (Fig. 3C) was mainly enriched in ion binding, receptor activity, identical protein binding and anion binding. The first 3 significantly enriched KEGG pathways (Fig. 3D) were cytokine-cytokine receptor interaction, thyroid cancer and prostate cancer.

PPI network analysis, and key nodes and pathway analyses in network. As shown in Fig. 4, there were 279 nodes and 575 protein pairs in the network. The first 12 nodes with the highest scores were taken from each centrality method, and 17 genes were obtained after merging and removing duplicates. The 17 genes were epidermal growth factor receptor (EGFR), cyclin D1 (CCND1), TIMP1, MMP1, PPARG, PTGS2, histone deacetylase 9 (HDAC9), IL-10, fms-related tyrosine kinase 1 (FLT1), heme oxygenase 1 (HMOX1), cyclin-dependent kinase inhibitor 1A (CDKN1A), Wnt family member 1 (WNT1), CD40 molecule (CD40), tensin 3 (TNS3), a2-macroglobulin (A2M), RAR-related orphan receptor $\mathrm{C}$ (RORC) and TF. The case samples and control samples were clustered by these 17 key genes. The centrality score of these 17 key genes is shown in Table II. As shown in Fig. 5, the pathways significantly enriched by these 17 genes were pathways in cancer, bladder cancer, cytokine-cytokine receptor interaction, and focal adhesion.

Transcription factors of key node analysis. As shown in Fig. 6, transcriptional regulation network included 27 nodes 
A

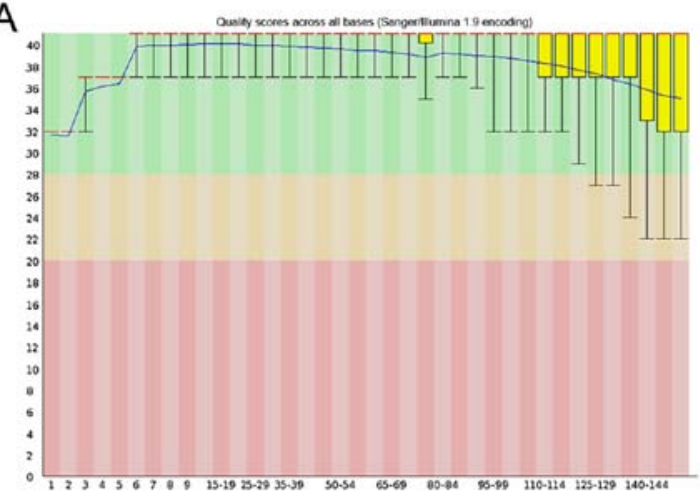

C

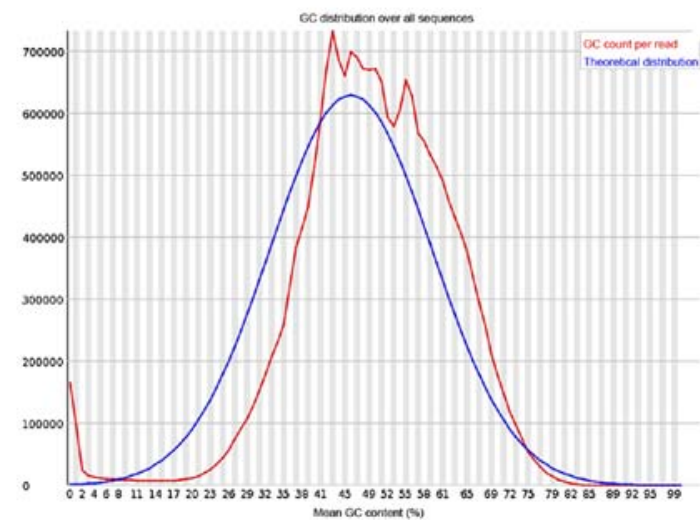

$\mathrm{B}$



D

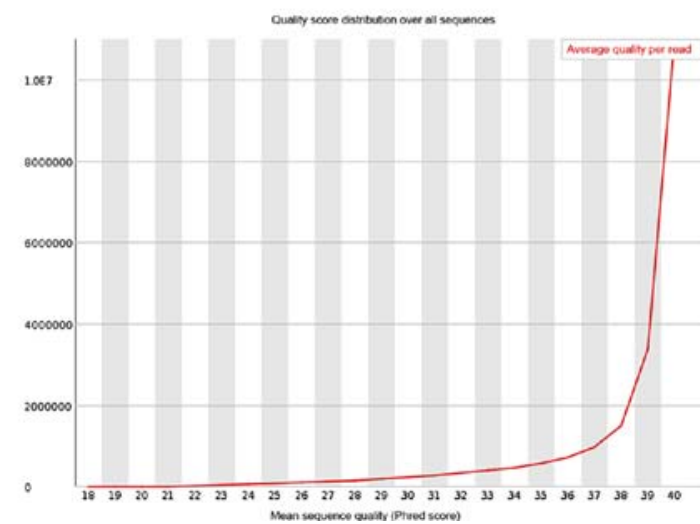

Figure 1. (A) The results for single base quality distribution, (B) base content distribution, (C) GC content distribution and (D) sequence base quality distribution. (A) Horizontal ordinate, position in reads (5'->3'); longitudinal coordinates, q-value statistics; red line represents, median; blue line, average number; yellow lines, the range of $25-75 \%$; tentacles, the range of $10-90 \%$. (B) Horizontal ordinate, position in reads (5'->3'); longitudinal coordinates, the proportion of a base. (C) Horizontal ordinate, mean GC content; longitudinal coordinates, the number of reads; red, actual distribution curve; blue, theoretical distribution curve. (D) Horizontal ordinate, quality (phred score); longitudinal coordinates, the number of reads.

A

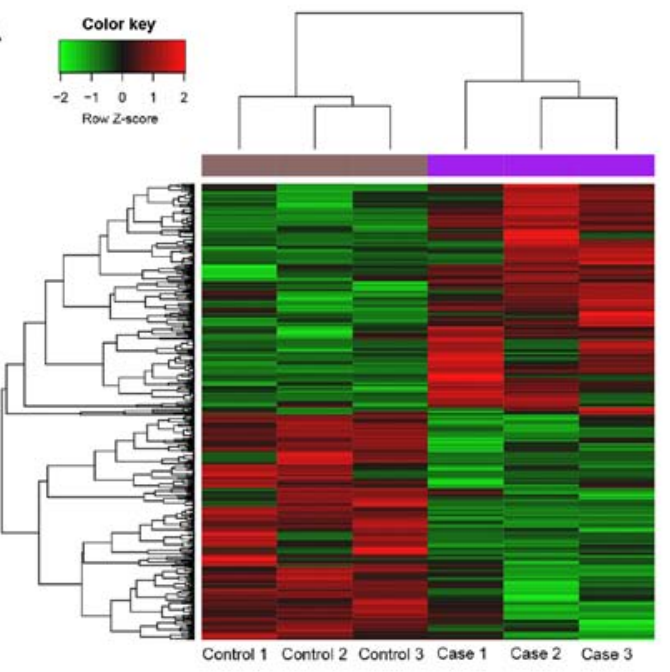

B

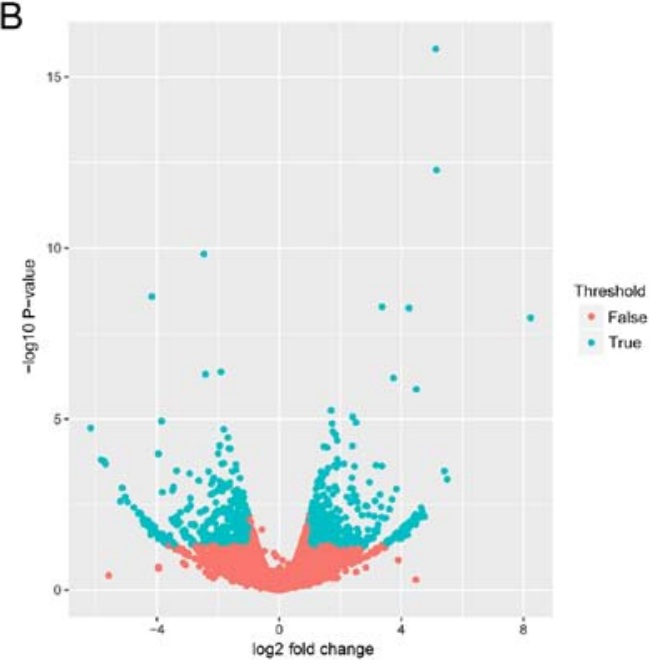

Figure 2. (A) Heat map of differentially expressed genes (DEGs). The green represents lower expression level; the red represents higher expression level; the black indicates that there is no differential expression among the gene. (B) Volcano plot.

and 78 interaction pairs. In total, 10 transcription factors were obtained with NES >3.5. TGIF2LY regulated MMP1, RORC, HDAC9, PPARG, WNT1, CCND1 and CDKN1A. SRF regulated MMP1, TNS3, A2M, CCND1 and CDKN1A. POU3F1 regulated CDKN1A, TNS3, WNT1, CCND1, HDAC9 and
PPARG. NKX2-2 regulated PPARG, CDKN1A, CCND1 and RORC. IRX4 regulated EGFR, PPARG, CCND1, WNT1 and CDKN1A. PRDM1 regulated RORC, TF, CDKN1A, A2M, HDAC9, CCND1 and CD40. HDX regulated 14 genes (all 17 genes except IL-10, PTGS2 and TF). PAX3 regulated FLT1, 

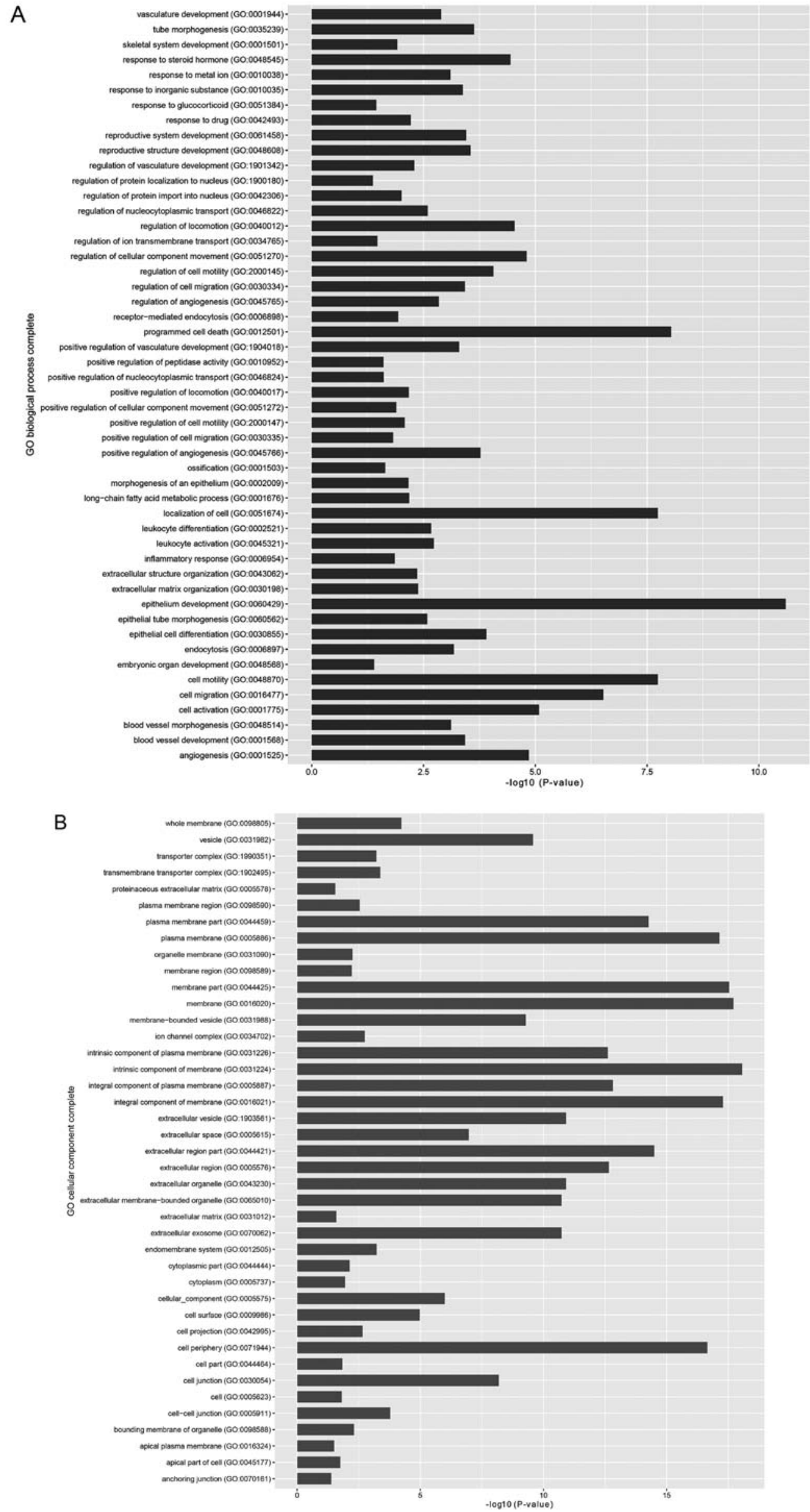

Figure 3. Gene Ontology (GO) slim and Kyoto Encyclopedia of Genes and Genomes (KEGG) pathway enrichment analyses for differentially expressed genes (DEGs). (A) GO-biological process (BP) (top 50) and (B) GO-cellular component (CC). 
C
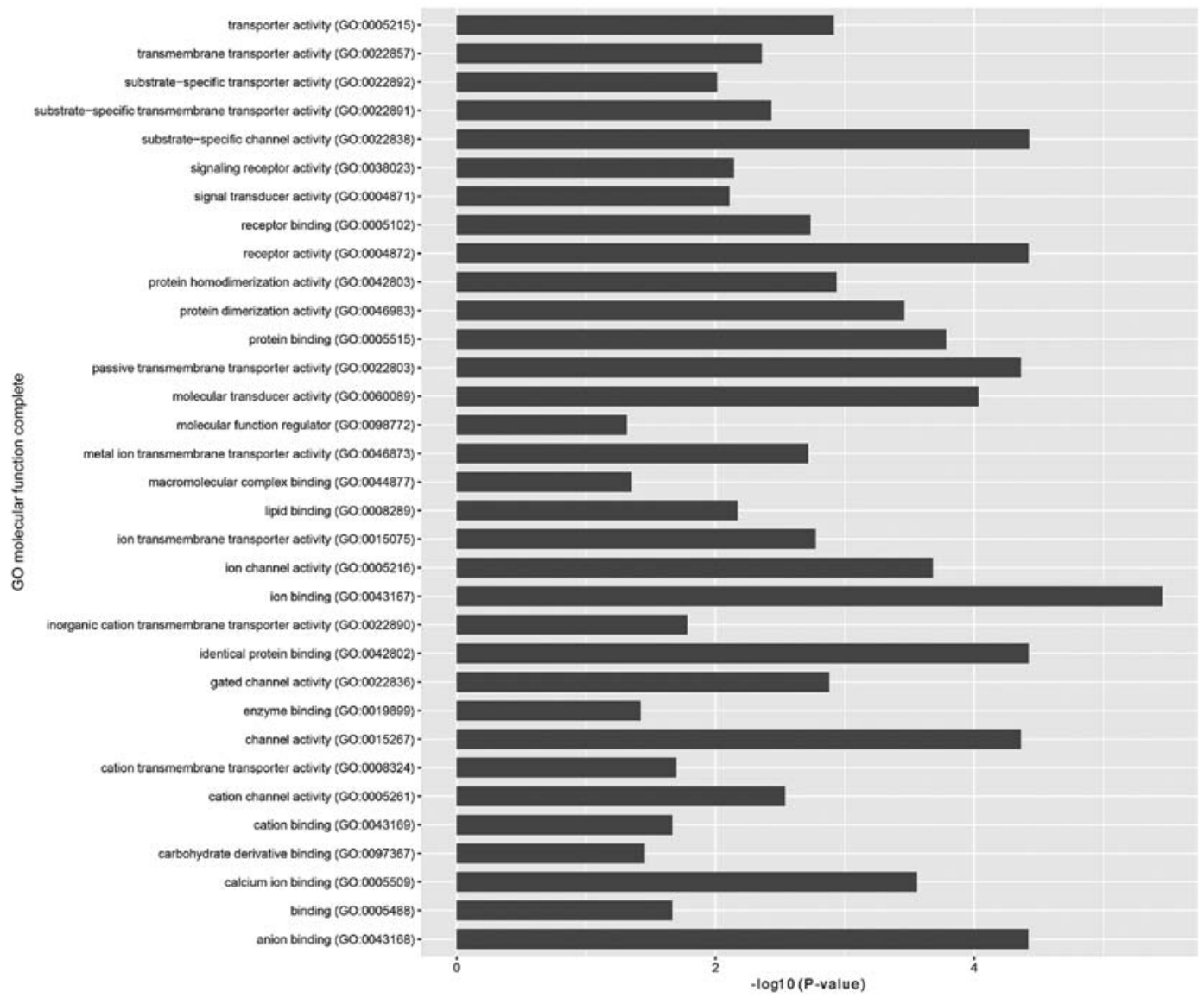

D

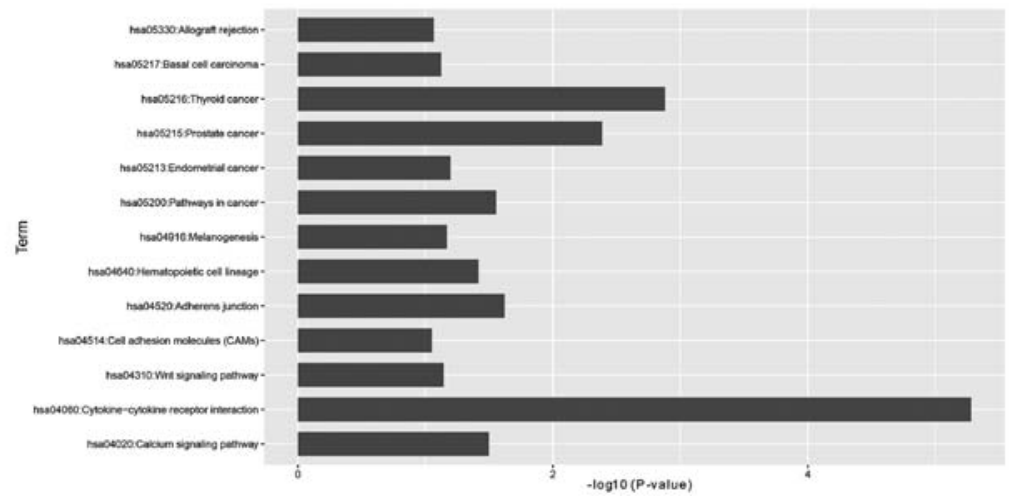

Figure 3. Continued. Gene Ontology (GO) slim and Kyoto Encyclopedia of Genes and Genomes (KEGG) pathway enrichment analyses for differentially expressed genes (DEGs). (C) GO-molecular function (MF) and (D) KEGG pathways.

CD40, TNS3, PPARG, CDKN1A, HDAC9 and HMOX1. FOS regulated 15 genes (all 17 genes except TF and PTGS2). CEBPG regulated CCND1, FLT1, EGFR, HDAC9, WNT1, PTGS2, PPARG and CDKN1A.

Verification of gene expression. The results of real-time PCR are shown in Fig. 7. In total 6 of 17 genes were detected. However, there was statistical significance between case and control groups for PTGS2, PPARG, TF and IL-10 ( $<<0.05)$, and there was no statistical significance between case and control groups for TIMP1 and MMP1 (Table III). All samples came from the same type of infections. It may be because the interference of external conditions leads to the unstable results. Thus, in our study, 6 samples were used as case and 6 samples for control.

\section{Discussion}

In the present study, in total 17 key genes in case group compared with the control group were identified by bioinformatics analysis. Then, 6 of 17 genes were detected by real-time PCR. However, there was statistical significance between case and control groups for PPARG, PTGS2, TF and IL-10 $(\mathrm{P}<0.05)$, and there was no statistical significance between case and control groups for TIMP1 and MMP1. 


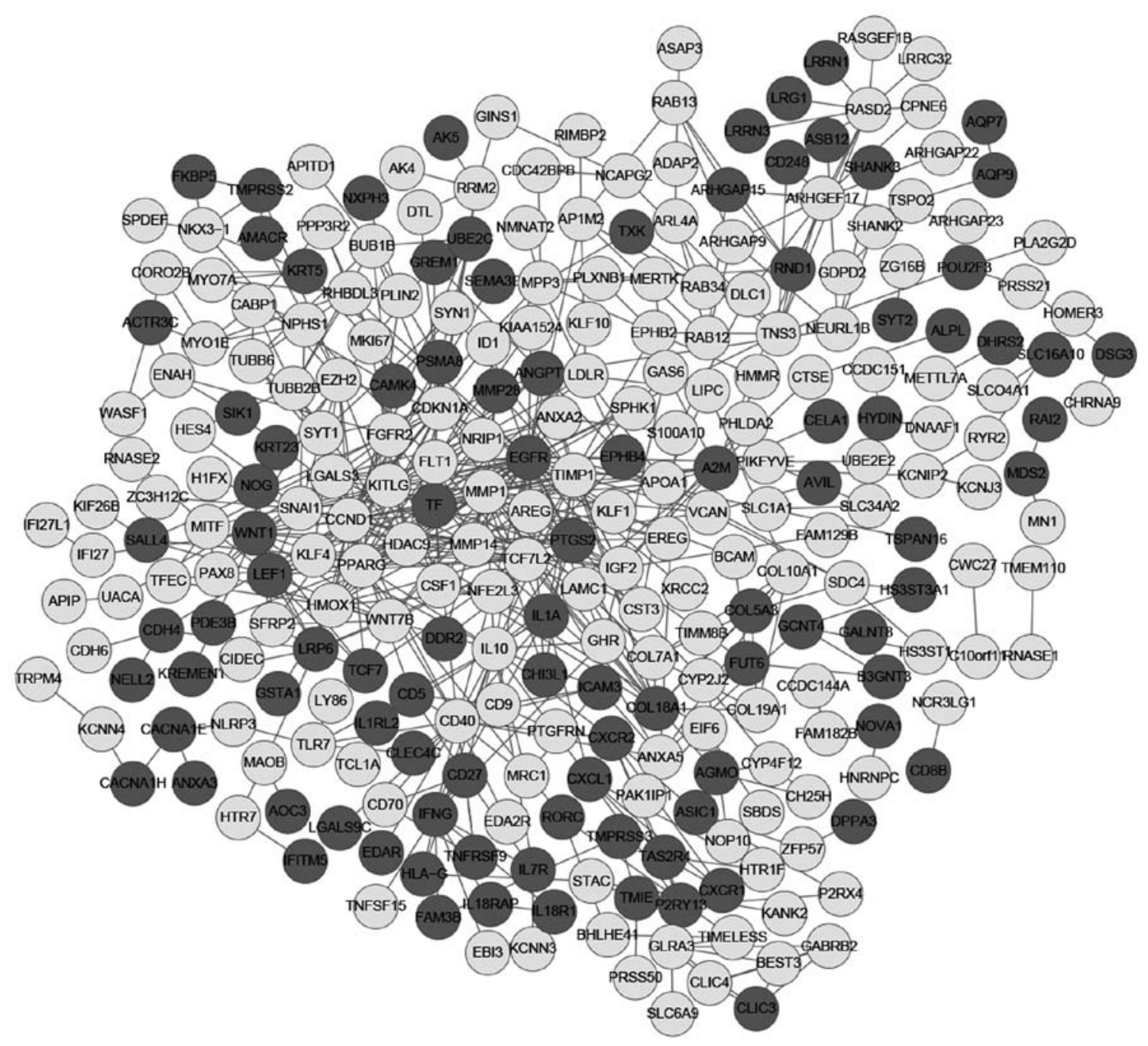

Figure 4. Protein-protein interaction (PPI) network of differentially expressed genes (DEGs). Light color nodes, downregulated genes; deep color nodes, upregulated genes.

Our study showed that PTGS2 and PPARG were key genes associated with RRTIs. Nuclear factor- $\kappa \mathrm{B}(\mathrm{NF}-\kappa \mathrm{B})$ is necessary for the roles of proinflammatory factors such as Cox-2 and IL-6 expression, and OM85-BV cause the translocation and activation of NF- $\mathrm{KB}$ (26). As previously mentioned, OM- 85 can decrease exacerbation frequency of respiratory tract infections in children and adults at risk (5). Furthermore, PTGS2 (Cox-2) mediates the anti-inflammatory response during Leishmania donovani infection (27). Cox-2, as a significant mediator, involves in respiratory syncytial virus-induced inflammation (28). In colorectal cancer, non-steroidal anti-inflammatory drugs can inhibit cancer stem cells through activating PPARG and suppressing PTGS2 and NOTCH/HES1 (29). Thus, PTGS2 may play important roles in the development of RRTIs. Besides, Bank et al indicated that PPARG involved in the regulation of inflammation (30). Malur et al suggested that PPARG played negative regulation roles for chronic granulomatous inflammation (31). Therefore, our results are in line with former research and show that PTGS2 and PPARG play significant parts in the progression of RRTIs.

Furthermore, bioinformatics analysis showed that TF was a key gene related with RRTIs, and it also was verified by real-time PCR in our present study. TF controls the free iron levels in biological fluids (32). TF is related with innate immune system. It can create low free iron environment and impede the survival of bacteria. The level of TF decrease in inflammation (33). Besides, a study showed that the injection of coenzyme A had important effect on the TF in elderly acute upper RTI patients (34). Thus, TF also play important roles in patients with RRTIs.

In addition, in the present study, IL-10 was also a key gene associated with RRTIs. Sun et al indicated that IL-10 played dual roles in immune response to respiratory syncytial virus (28). On the one hand, IL-10 inhibits respiratory syncytial virus induced inflammation; on the other hand, it induces the Th2-dominant immune responses. Furthermore, in physically active individuals, high IL-10 is a risk factor for the progression of upper RTIs (35). Besides, Bont et al indicated that increased production of IL-10 was related with the development of recurrent wheezing after respiratory syncytial virus bronchiolitis (36). Thus, our results are in accord with the former studies and suggest that IL-10 is associated with the development of RRTIs.

In this study, the bioinformatics analysis identified 17 genes, and 6 of these genes including PPARG, PTGS2, TF, IL-10, 


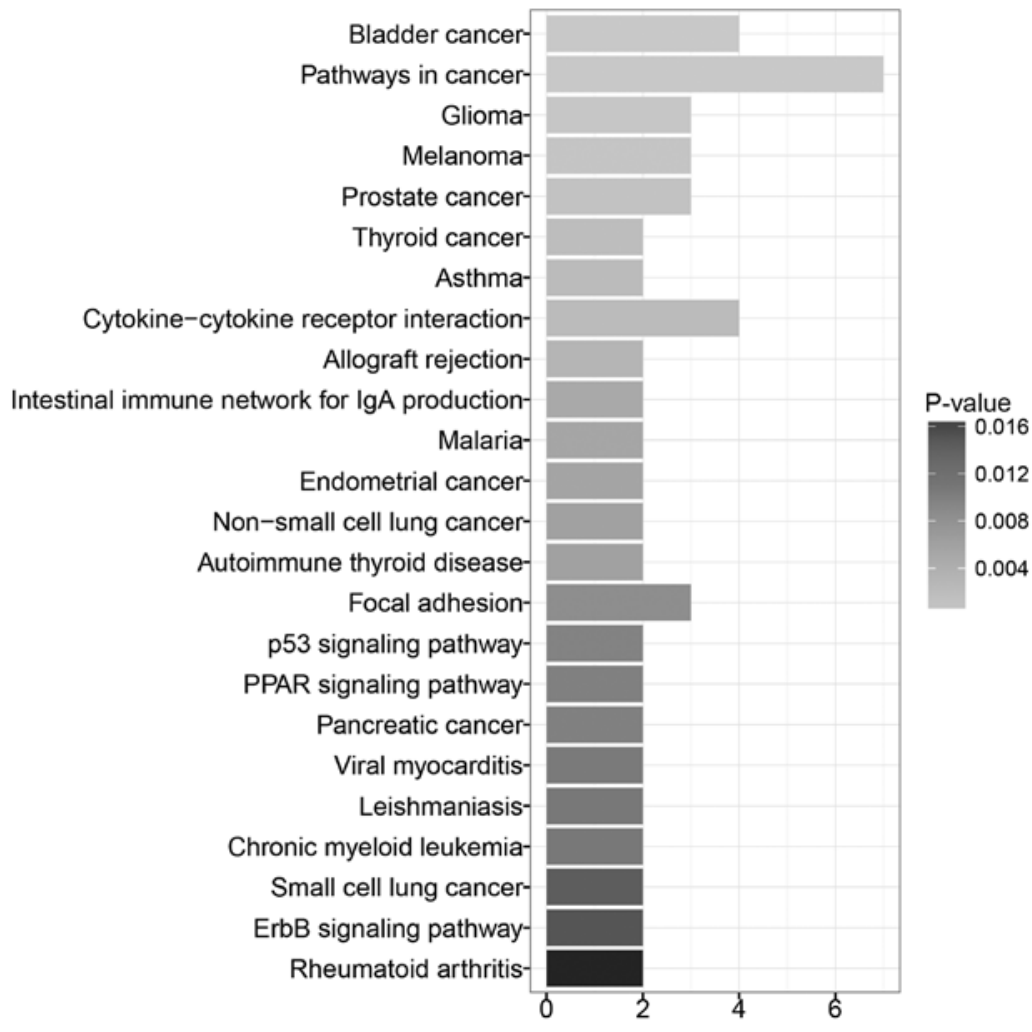

Figure 5. The pathways significantly enriched by 17 genes.

Table II. The centrality score of 17 key genes.

\begin{tabular}{lrrrr}
\hline Genes & Subgragh & Degree & Betweenness & Closeness \\
\hline EGFR & 41129.95 & 41 & 21125.9 & 0.035257 \\
CCND1 & 29876.16 & 31 & 8902.149 & 0.035039 \\
TIMP1 & 23954.49 & 25 & 3898.972 & 0.034938 \\
MMP1 & 22258.21 & 24 & 3890.898 & 0.034955 \\
PPARG & 19492.27 & 25 & 10108.1 & 0.035021 \\
PTGS2 & 10549.89 & 15 & 3224.803 & 0.034815 \\
HDAC9 & 10406.45 & 20 & 5808.442 & 0.034767 \\
IL-10 & 9644.303 & 20 & 10592.6 & 0.034881 \\
FLT1 & 8960.905 & 17 & 2048.353 & 0.034612 \\
HMOX1 & 8760.095 & 13 & 1930.259 & 0.034802 \\
CDKN1A & 8346.486 & 18 & 4548.326 & 0.034711 \\
WNT1 & 7728.413 & 15 & 1505.885 & 0.034599 \\
CD40 & 5181.088 & 19 & 7817.111 & 0.034828 \\
TNS3 & 358.5152 & 10 & 7723.212 & 0.034466 \\
A2M & 2485.854 & 11 & 5437.33 & 0.034478 \\
RORC & 129.5831 & 3 & 3904 & 0.034014 \\
TF & 6344.649 & 11 & 841.7473 & 0.034707 \\
\hline
\end{tabular}

EGFR, epidermal growth factor receptor; CCND1, cyclin D1; TIMP1, TIMP metallopeptidase inhibitor 1; MMP1, matrix metallopeptidase 1; PPARG, peroxisome proliferator-activated receptor- $\gamma$; PTGS2, prostaglandin-endoperoxide synthase 2; HDAC9, histone deacetylase 9; IL-10, interleukin-10; FLT1, fms-related tyrosine kinase 1; HMOX1, heme oxygenase 1; CDKN1A, cyclin-dependent kinase inhibitor 1A; WNT1, Wnt family member 1; CD40, CD40 molecule; TNS3, tensin 3; A2M, $\alpha 2$-macroglobulin; RORC, RAR-related orphan receptor $\mathrm{C}$.
Table III. The result of statistical analysis for verification of gene expression.

\begin{tabular}{lcccccrrrr}
\hline & \multicolumn{2}{c}{ Case group } & & \multicolumn{2}{c}{ Control group } & & \\
\cline { 2 - 3 } Item & $\mathrm{N}$ & mean \pm SEM & & $\mathrm{N}$ & mean \pm SEM & & t-test & P-value \\
\hline TIMP1 & 18 & $1.068 \pm 0.305$ & 18 & $1.068 \pm 0.337$ & 0.001 & 0.999 \\
MMP1 & 18 & $16.581 \pm 24.360$ & & 18 & $11.560 \pm 5.973$ & 0.849 & 0.406 \\
PPARG & 17 & $1.347 \pm 0.452$ & & 18 & $5.738 \pm 2.405$ & 7.605 & $<0.001$ \\
PTGS2 & 18 & $0.745 \pm 0.341$ & & 18 & $0.311 \pm 0.173$ & 4.820 & $<0.001$ \\
TF & 18 & $1.239 \pm 0.524$ & & 18 & $0.744 \pm 0.459$ & 3.020 & 0.005 \\
IL-10 & 18 & $1.539 \pm 0.682$ & & 18 & $3.452 \pm 2.877$ & 2.744 & 0.013
\end{tabular}

$\mathrm{P}<0.05$ was considered to be statistically significant. TIMP1, TIMP metallopeptidase inhibitor 1; MMP1, matrix metallopeptidase 1; PPARG, peroxisome proliferator-activated receptor- $\gamma$; PTGS2, prostaglandin-endoperoxide synthase 2 ; TF, transferrin; IL-10, interleukin-10.

TIMP1 and MMP1 were detected by real-time PCR. But there was no statistical significance between case and control groups for TIMP1 and MMP1. Besides, previous studies showed that MMPs and their tissue inhibitors of TIMP1 and TIMP2 were involved in tissue remodeling during inflammation (37-39). We speculate that TIMP1 and MMP1 also may be involved in the progression of RRTIs. But, further studies with large number of samples are needed for verification.

The obvious advantage of this study is that the results of bioinformatics analysis are verified by real-time PCR. However, the disadvantage is that several genes identified 


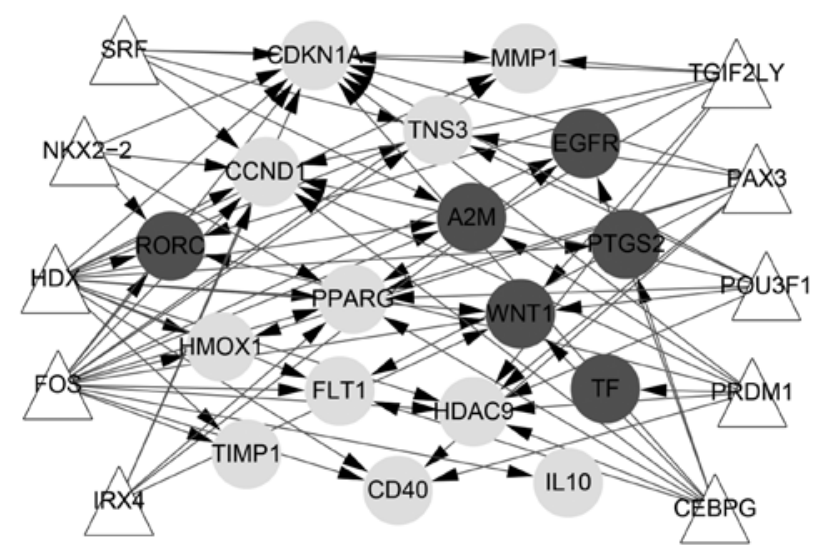

Figure 6. Transcriptional regulation network of key genes. Light color nodes, downregulated genes; deep color nodes, upregulated genes; triangle, transcription factor.


Figure 7. The results of real-time PCR for TIMP metallopeptidase inhibitor 1 (TIMP1), matrix metallopeptidase 1 (MMP1), peroxisome proliferator-activated receptor- $\gamma$ (PPARG), prostaglandin-endoperoxide synthase 2 (PTGS2), transferrin (TF) and interleukin-10 (IL-10). (A) MMP1; (B) TIMP1; (C) PTGS2 $(\mathrm{P}<0.05)$; (D) PPARG $(\mathrm{P}<0.05)$; (E) TF $(\mathrm{P}<0.05)$ and $(\mathrm{F})$ IL-10 $(\mathrm{P}<0.05)$.

by bioinformatics are not verified by real-time PCR possibly because of the small sample size.
In conclusion, PPARG, PTGS2, TF and IL-10 are key genes associated with the progression of RRTIs. PPARG, 
PTGS2, TF and IL-10 may be regarded as a therapeutic target of RRTIs. We speculate that TIMP1 and MMP1 may also be involved in the progression of RRTIs, but further studies with large number of samples are needed for verification.

\section{Acknowledgements}

Not applicable.

\section{Funding}

No funding was received.

\section{Availability of data and material}

The datasets used and/or analyzed during the current study are available from the corresponding author on reasonable request.

\section{Authors' contributions}

YG and XJ contributed to the conception and design of the research and drafting of the manuscript. $\mathrm{ZJ}$ contributed to the acquisition of data. RL analyzed and interpreted the data. XL performed the statistical analysis. YG revised the manuscript for important intellectual content. All authors read and approved the final manuscript.

\section{Ethics approval and consent to participate}

The present study was approved by Ethics Committee of First Hospital, Jilin University (no. 2014-078). Single RNA sample was not up to the requirements of sequencing, thus 3 cases of blood samples were extracted and mixed into 1 sample for sequencing.

\section{Consent for publication}

Not applicable.

\section{Competing interests}

The authors declare that they have no competing interests.

\section{References}

1. Principi N, Esposito S, Cavagna R, Bosis S, Droghetti R, Faelli N, Tosi S and Begliatti E; Snoopy Study Group: Recurrent respiratory tract infections in pediatric age: a population-based survey of the therapeutic role of macrolides. J Chemother 15 53-59, 2003

2. de Martino M and Ballotti S: The child with recurrent respiratory infections: normal or not? Pediatr Allergy Immunol 18 (Suppl 18): 13-18, 2007.

3. Bellanti JA: Recurrent respiratory tract infections in paediatric patients. Drugs 54 (Suppl 1): 1-4, 1997.

4. Khasawneh FA and Jou-Tindo AJ: A 30-year-old woman with recurrent lower respiratory tract infections. Chest 143 : 1500-1503, 2013.

5. De Benedetto F and Sevieri G: Prevention of respiratory tract infections with bacterial lysate OM-85 bronchomunal in children and adults: a state of the art. Multidiscip Respir Med 8: 33, 2013

6. Bergman P, Norlin A-C, Hansen S, Rekha RS, Agerberth B, Björkhem-Bergman L, Ekström L, Lindh JD and Andersson J: Vitamin $\mathrm{D}_{3}$ supplementation in patients with frequent respiratory tract infections: a randomised and double-blind intervention study. BMJ Open 2: e001663, 2012.
7. Jesenak M, Hrubisko M, Majtan J, Rennerova $Z$ and Banovcin P: Anti-allergic effect of Pleuran ( $\beta$-glucan from Pleurotus ostreatus) in children with recurrent respiratory tract infections. Phytother Res 28: 471-474, 2014.

8. Maldonado J, Cañabate F, Sempere L, Vela F, Sánchez AR, Narbona E, López-Huertas E, Geerlings A, Valero AD, Olivares M, et al: Human milk probiotic Lactobacillus fermentum CECT5716 reduces the incidence of gastrointestinal and upper respiratory tract infections in infants. J Pediatr Gastroenterol Nutr 54: 55-61, 2012.

9. Orlowsky EW and Kraus VB: The role of innate immunity in osteoarthritis: when our first line of defense goes on the offensive. J Rheumatol 42: 363-371, 2015.

10. Martin M: Cutadapt removes adapter sequences from highthroughput sequencing reads. EMBnet 17: 10-12, 2011.

11. Ritchie ME, Phipson B, Wu D, Hu Y, Law CW, Shi W and Smyth GK: Limma powers differential expression analyses for RNA-sequencing and microarray studies. Nucleic Acids Res 43: e47, 2015.

12. Smyth GK: LIMMA: linear models for microarray data. In: Statistics for Biology and Health: Bioinformatics and Computational Biology Solutions Using R and Bioconductor. Springer, New York, pp397-420, 2005.

13. Law CW, Chen Y, Shi W and Smyth GK: voom: Precision weights unlock linear model analysis tools for RNA-seq read counts. Genome Biol 15: R29, 2014.

14. Warnes GR, Bolker B, Bonebakker L, Gentleman R, Liaw A, Lumley T, Maechler M, Magnusson A, Moeller S, Schwartz M, et al: gplots: Various R programming tools for plotting data. $R$ package version 2: 2009 .

15. Ashburner M, Ball CA, Blake JA, Botstein D, Butler H, Cherry JM, Davis AP, Dolinski K, Dwight SS, Eppig JT, et al; The Gene Ontology Consortium: Gene ontology: tool for the unification of biology. Nat Genet 25: 25-29, 2000.

16. Szklarczyk D, Franceschini A, Wyder S, Forslund K, Heller D, Huerta-Cepas J, Simonovic M, Roth A, Santos A, Tsafou KP, et al: STRING v10: protein-protein interaction networks, integrated over the tree of life. Nucleic Acids Res 43: D447-D452, 2015.

17. Shannon P, Markiel A, Ozier O, Baliga NS, Wang JT, Ramage D, Amin N, Schwikowski B and Ideker T: Cytoscape: a software environment for integrated models of biomolecular interaction networks. Genome Res 13: 2498-2504, 2003.

18. Opsahl T, Agneessens F and Skvoretz J: Node centrality in weighted networks: generalizing degree and shortest paths. Soc Networks 32: 245-251, 2010.

19. Wang H, Hernandez JM and Van Mieghem P: Betweenness centrality in a weighted network. Phys Rev E Stat Nonlin Soft Matter Phys 77: 046105, 2008.

20. Estrada E and Rodríguez-Velázquez JA: Subgraph centrality in complex networks. Phys Rev E Stat Nonlin Soft Matter Phys 71: 056103, 2005.

21. Okamoto K, Chen W and Li XY: Ranking of closeness centrality for large-scale social networks. In: Frontiers in Algorithmics. Springer, Berlin, pp186-195, 2008.

22. Tang Y, Li M, Wang J, Pan Y and Wu FX: CytoNCA: a cytoscape plugin for centrality analysis and evaluation of protein interaction networks. Biosystems 127: 67-72, 2015.

23. Yu G, Wang LG, Han Y and He QY: clusterProfiler: an R package for comparing biological themes among gene clusters. OMICS 16: 284-287, 2012 .

24. Ferreira JA: The Benjamini-Hochberg method in the case of discrete test statistics. Int J Biostat 3: 11, 2007.

25. Janky R, Verfaillie A, Imrichová H, Van de Sande B, Standaert L, Christiaens V, Hulselmans G, Herten K, Naval Sanchez M, Potier D, et al: iRegulon: from a gene list to a gene regulatory network using large motif and track collections. PLOS Comput Biol 10: e1003731-e1003731, 2014.

26. Luan H, Zhang Q, Wang L, Wang C, Zhang M, Xu X, Zhou H, Li X, $\mathrm{Xu} \mathrm{Q}, \mathrm{He} \mathrm{F}$, et al: OM85-BV induced the productions of IL-1 $\beta$, IL-6, and TNF- $\alpha$ via TLR4- and TLR2-mediated ERK1/2/NF- $\kappa$ B pathway in RAW264.7 cells. J Interferon Cytokine Res 34: 526-536, 2014.

27. Bhattacharjee S, Bhattacharjee A, Majumder S, Majumdar SB and Majumdar S: Glycyrrhizic acid suppresses Cox-2-mediated anti-inflammatory responses during Leishmania donovani infection. J Antimicrob Chemother 67: 1905-1914, 2012.

28. Sun L, Cornell TT, LeVine A, Berlin AA, Hinkovska-Galcheva V, Fleszar AJ,Lukacs NW and Shanley TP:Dual role of interleukin-10 in the regulation of respiratory syncitial virus (RSV)-induced lung inflammation. Clin Exp Immunol 172: 263-279, 2013. 
29. Moon CM, Kwon JH, Kim JS, Oh SH, Jin Lee K, Park JJ, Pil Hong S, Cheon JH, Kim TI and Kim WH: Nonsteroidal anti-inflammatory drugs suppress cancer stem cells via inhibiting PTGS2 (cyclooxygenase 2) and NOTCH/HES1 and activating PPARG in colorectal cancer. Int J Cancer 134: 519-529, 2014.

30. Bank S, Skytt Andersen P, Burisch J, Pedersen N, Roug S, Galsgaard J, Ydegaard Turino S, Brodersen JB, Rashid S, Kaiser Rasmussen B, et al: Polymorphisms in the inflammatory pathway genes TLR2, TLR4, TLR9, LY96, NFKBIA, NFKB1, TNFA, TNFRSF1A, IL6R, IL10, IL23R, PTPN22, and PPARG are associated with susceptibility of inflammatory bowel disease in a Danish cohort. PLoS One 9: e98815-e98815, 2014.

31. Huizar I, Malur A, Patel J, McPeek M, Dobbs L, Wingard C, Barna BP and Thomassen MJ: The role of PPAR $\gamma$ in carbon nanotube-elicited granulomatous lung inflammation. Respir Res 14: 7, 2013.

32. Crichton RR and Charloteaux-Wauters M: Iron transport and storage. Eur J Biochem 164: 485-506, 1987.

33. Ritchie RF, Palomaki GE, Neveux LM, Navolotskaia O, Ledue TB and Craig WY: Reference distributions for the negative acute-phase serum proteins, albumin, transferrin and transthyretin: a practical, simple and clinically relevant approach in a large cohort. J Clin Lab Anal 13: 273-279, 1999.
34. Wang Q and Bai Q: Influence of injection with coenzyme A on IL-8, C-reaction protein, transferrin in the elderly with acute upper respiratory tract infections. Chinese J Nosocomiol 15: 009, 2013 (In Chinese).

35. Gleeson M, Bishop N, Oliveira M, McCauley T, Tauler P and Muhamad AS: Respiratory infection risk in athletes: association with antigen-stimulated IL-10 production and salivary IgA secretion. Scand J Med Sci Sports 22: 410-417, 2012.

36. Bont L, Heijnen CJ, Kavelaars A, van Aalderen WM, Brus F, Draaisma JT, Geelen SM and Kimpen JL: Monocyte IL-10 production during respiratory syncytial virus bronchiolitis is associated with recurrent wheezing in a one-year follow-up study. Am J Respir Crit Care Med 161: 1518-1523, 2000.

37. Tryggvason K, Höyhtyä M and Salo T: Proteolytic degradation of extracellular matrix in tumor invasion. Biochim Biophys Acta 907: 191-217, 1987.

38. Karelina TV, Hruza GJ, Goldberg GI and Eisen AZ: Localization of 92-kDa type IV collagenase in human skin tumors: comparison with normal human fetal and adult skin. J Invest Dermatol 100: 159-165, 1993.

39. Woessner JF Jr: Matrix metalloproteinases and their inhibitors in connective tissue remodeling. FASEB J 5: 2145-2154, 1991. 